\title{
Prognostic value of regional strain by cardiovascular magnetic resonance feature tracking in hypertrophic cardiomyopathy
}

\author{
Xiaorong Chen ${ }^{1,2 \#}$, Jiangfeng Pan ${ }^{1 \#}$, Jiner Shu ${ }^{3}$, Xiaoru Zhang ${ }^{1}$, Limei Ye $^{1}$, Lin Chen ${ }^{1}$, Yi Hu ${ }^{1}$, Risheng Yu ${ }^{2}$ \\ ${ }^{1}$ Department of Medical Imaging, Affiliated Jinhua Hospital, Zhejiang University School of Medicine, Jinhua, China; ${ }^{2}$ Department of Radiology, The \\ Second Affiliated Hospital, Zhejiang University School of Medicine, Hangzhou, China; ${ }^{3}$ Department of Radiology, Jinhua People's Hospital, Jinhua, \\ China
}

Contributions: (I) Conception and design: R Yu; (II) Administrative support: J Shu; (III) Provision of study materials or patients: J Pan; (IV) Collection and assembly of data: X Zhang, L Ye, L Chen, Y Hu; (V) Data analysis and interpretation: X Chen; (VI) Manuscript writing: All authors; (VII) Final approval of manuscript: All authors.

\#These authors contributed equally to this work.

Correspondence to: Risheng Yu. Department of Radiology, The Second Affiliated Hospital, Zhejiang University School of Medicine, No. 88 Jiefang Road, Hangzhou 310009, China. Email: risheng-yu@zju.edu.cn; Jiner Shu. Department of Radiology, Jinhua People’s Hospital, No. 267 East Danxi Road, Jinhua 321000, China. Email: shujiner@163.com.

Background: Few studies have demonstrated the performance of regional strain by cardiovascular magnetic resonance (CMR) feature tracking in hypertrophic cardiomyopathy (HCM) patients, and the prognostic value of segmental strain remains unknown. This study aimed to explore the prognostic implications of strain parameters generated by CMR feature tracking analysis in HCM patients.

Methods: In total, 104 clinically diagnosed HCM patients and 30 healthy volunteers were enrolled in this study, and all patients underwent a standard CMR examination. Global and regional strain was computed by short axis, 2-, 3-, and 4-chamber view cine MR imaging using specialized software. Cardiac structure, function, and myocardial strain were compared between the control group and HCM patients, and the event and event-free groups. Univariate and multivariate Cox regression analyses were performed to evaluate the correlations between clinical and CMR parameters and poor prognosis.

Results: During the follow-up time, 8 patients reached the primary end points and 14 patients reached secondary end points. Regional radial strain of hypertrophic segments (RRS) and regional circumferential strain of hypertrophic segments (RCS) were worse in HCM patients with primary and secondary end points. In univariate Cox regression analysis of RRS, RCS were associated with primary and secondary end points. Regional radial strain of hypertrophic segments [hazard ratio (HR) 1.64, 95\% confidence interval (CI): 1.132.38] and RCS (HR 2.35, 95\% CI: 1.20-4.59) were independent predictors of primary end points, and RRS (HR 1.71, 95\% CI: 1.09-2.66) and RCS (HR 2.63, 95\% CI: 1.20-5.75) remained independent predictors of secondary end points in multivariate analysis. Kaplan-Meier survival curves indicated patients with RRS $<10.0 \%$ and $\mathrm{RCS} \geq-8.5 \%$ had a higher rate of primary end points, and patients with RRS $<17.9 \%$ and RCS $\geq-12.1 \%$ experienced a higher rate of secondary end points.

Conclusions: In HCM patients, RRS and RCS were associated with primary and secondary end points and remained independent predictors in multivariate analysis. Impaired regional strain may potentially predict poor prognosis in HCM patients.

Keywords: Prognosis; hypertrophic cardiomyopathy (HCM); cardiovascular magnetic resonance (CMR); regional strain 
Submitted Jan 11, 2021. Accepted for publication Jul 21, 2021.

doi: 10.21037/qims-21-42

View this article at: https://dx.doi.org/10.21037/qims-21-42

\section{Introduction}

Hypertrophic cardiomyopathy (HCM) is the most common genetic cardiomyopathy and is usually defined by increased ventricular wall thickness with non-dilated ventricular chamber, which cannot be explained by loading conditions. In general adult samples, the prevalence of HCM is reported to be approximately $0.02-0.23 \%(1,2)$. HCM is usually clinically diagnosed by maximal left ventricular (LV) wall thickness $\geq 15 \mathrm{~mm}$ in at least $1 \mathrm{LV}$ myocardial segment based on echocardiography, cardiovascular magnetic resonance (CMR) imaging, or cardiac computed tomography $(1,3)$.

HCM is one of the most common causes of sudden cardiac death (SCD). The annual incidence of SCD, heart failure, and stroke is about $1-2 \%(4,5)$. Risk factors for SCD in HCM patients include non-sustained ventricular arrhythmia, $\mathrm{LV}$ wall thickness (LVWT) $\geq 30 \mathrm{~mm}$, prior history and family history of SCD, and unexplained syncope $(1,4)$. Restrictive filling pattern, $\mathrm{LV}$ apical aneurysms, atrial fibrillation, left ventricular ejection fraction (LVEF), left atrial (LA) size, ejection fraction (LAEF), and late gadolinium enhancement (LGE) (6-11) are also likely to be associated with a higher risk of a major adverse cardiovascular event.

Myocardial strain is a non-invasive tool to evaluate ventricular function, especially to detect ventricular dysfunction in preserved ejection function (EF) in HCM patients (12-14). HCM-based feature tracking imaging is a new technique for strain analysis and has evolved rapidly in recent years. It has been proven to be robust with good reproducibility and agreement with CMR tagging (15). Previous studies have also reported the prognostic value of global strain aside from its diagnostic value, and an abnormal global longitudinal strain has been found to be associated with adverse composite cardiac outcomes and ventricular arrhythmias (16). However, global strain cannot indicate regional function, whereas regional strain can do so. A few research studies have examined the diagnostic value of regional strain $(17,18)$ and described impaired segmental strain in HCM patients; however, the prognostic value of using CMR feature tracking imaging is still unknown. In this study, we aimed to explore the prognostic implications of strain parameters generated by CMR feature tracking analysis in HCM patients.

\section{Methods}

\section{Patient population}

This study consecutively included 104 clinically diagnosed HCM patients evaluated in our department between March 2013 and May 2019. The diagnostic criteria followed the 2011 American Heart Association (AHA) and 2014 European Society of Cardiology (ESC) guidelines $(1,4)$ on diagnosis and management of HCM. HCM is clinically diagnosed when maximal LVWT $\geq 15 \mathrm{~mm}$ or with a borderline wall thickness of 13 to $14 \mathrm{~mm}$ based on CMR in the presence of a family history of HCM. Obstructive HCM is confirmed when the $\mathrm{LV}$ outflow tract or mid $\mathrm{LV}$ cavity peak gradient are $\geq 30 \mathrm{mmHg}$ at rest. Apical HCM is confirmed when the apical to basal LVWT ratio is $\geq 1.3-1.5$ (3). Thirty age- and gender-matched healthy volunteers were enrolled as the control group. The study was conducted in accordance with the Declaration of Helsinki (as revised in 2013). The study was approved by the ethics committee of our institute, and written informed consent was obtained from each patient and volunteer.

\section{CMR protocol}

One 1.5 T MRI system (Avanto, Siemens, Munich, Germany) and one 3.0 T MRI system (Achieva, Philips, Eindhoven, The Netherlands) were used to obtain MRI images. MRI acquisition on both systems included 2-, 3 -, and 4-chamber and short axis (SA) view cine MR imaging and LGE images. LGE delay time (10 to $15 \mathrm{~min}$ ) and injected contrast dose $(0.2 \mathrm{mmol} / \mathrm{kg}$ gadodiamide, Omniscan, GE Healthcare, Cork, Ireland) were also the same between patients. The coverage of the SA view was the whole left ventricle from the base to apex. Cine MR imaging was performed using retrospective electrocardiogram (ECG) gating, LGE images were acquired using ECG triggering, and both were performed with breath holding in a supine position. An 8-channel phased-array body coil was used in a $1.5 \mathrm{~T}$ Siemens system. A true fast cine MR imaging sequence with steady precession was used with the following parameters: slice thickness $=8 \mathrm{~mm}$, gap $=2 \mathrm{~mm}$, field of view $(\mathrm{FOV})=330 \mathrm{~mm} \times 280 \mathrm{~mm}$, repetition time $(\mathrm{TR})=39.75 \mathrm{~ms}$, echo time $(\mathrm{TE})=1.12 \mathrm{~ms}$, flip angle $=42^{\circ}$, and reconstructed 
cardiac phases $=25$. LGE imaging consisted of magnitude and phase-sensitive inversion recovery (PSIR) images, with the following parameters: $\mathrm{TR}=700 \mathrm{~ms}, \mathrm{TE}=3.36 \mathrm{~ms}$, flip angle $=25^{\circ}$, inversion time $(\mathrm{TI})=200-300 \mathrm{~ms}$; slice thickness $=8 \mathrm{~mm}$, and gap $=2 \mathrm{~mm}$. A 16-channel dedicated phased-array torso coil was used in a 3.0-T system. The cine MR imaging based on sense balanced turbo field echo sequence was used, with FOV $=300 \times 300 \mathrm{~mm}$, TR/TE $=2.89 / 1.45 \mathrm{~ms}$, flip angle $=45^{\circ}$, reconstructed cardiac phases $=25$, slice thickness $=8 \mathrm{~mm}$, and gap $=2 \mathrm{~mm}$. LGE images used a phase-sensitive inversion recovery turbo field echo (PSIR-TFE) sequence, with FOV $=300 \times 300 \mathrm{~mm}$, TR/ $\mathrm{TE}=6.12 / 3.00 \mathrm{~ms}$, $\mathrm{TI}=280-450 \mathrm{~ms}$, flip angle $=25^{\circ}$, slice thickness $=8 \mathrm{~mm}$, and gap $=2 \mathrm{~mm}$. A nonenhanced CMR protocol was performed for healthy volunteers, using a 3.0-T Philips system with a 16-channel dedicated phasedarray torso coil and the same scanning parameters.

\section{CMR analysis}

CMR analysis was performed using specialized software (CVI 42, version 5.11.3, Circle, Calgary, Canada), consisting of LVWT, biventricular, left atrial structure and function, and LGE quantification. Left ventricular wall thickness was obtained via directly measuring the maximum thickened wall thickness at end diastole. Biventricular structure and function analysis were based on SA view cine MR imaging. The endocardium and epicardium were contoured manually, slice by slice, at end systolic and end diastolic phases. The papillary muscle was included in LV volume and excluded from the endocardial ventricular border (19). Trabeculations of the LV and right ventricular (RV) were ignored and included in ventricular volume. After contouring was completed, ventricular structural and functional indices were generated according to the Simpson method, including left ventricular end diastolic volume (LVEDV), left ventricular end systolic volume (LVESV), LVEF, mass, right ventricular end diastolic volume (RVEDV), right ventricular end systolic volume (RVESV), and right ventricular ejection fraction (RVEF).

Left atrial volume (LAV) and function analysis were based on the biplane (2- and 4-chamber view cine MR imaging) area-length method, and endocardial contouring was performed manually at phases when the left atrium was maximum and minimum. Left atrial volume was calculated according the following formula (20): LAV $=0.85 \times \mathrm{A} 2 \mathrm{C} \times \mathrm{A} 4 \mathrm{C} / \mathrm{L} . \mathrm{A} 2 \mathrm{C}$, where $\mathrm{A} 4 \mathrm{C}$ denotes the LA areas on the 2 - and 4 -chamber views, and $\mathrm{L}$ is the shorter long-axis length of the LA from these 2 views. Left atrial maximum volume (LAVmax), left atrial minimum volume (LAVmin), and LAEF were calculated.

LGE quantification was performed using a tissue characterization module. The SA view of PSIR images was used to quantify LGE, while 2-, 3-, and 4-chamber views were used to help distinguish the enhancement from the artefact. LGE was calculated using a semiquantitative analysis, according to the following formula: threshold $=$ mean +6 standard deviation (21), where mean and standard deviation were generated when a region of interest approximately $20 \mathrm{~mm}^{2}$ was placed at the remote myocardium. The area with a higher intensity than threshold was deemed an LGE area. Finally, an experienced observer (with more than 10 years of experience in CMR) adjusted the LGE area if an artefact was included.

\section{Feature tracking analysis}

Feature tracking analysis was performed using CVI 42 software (Circle Cardiovascular Imaging). In tissue tracking module, SA, 2-, 3- and 4-chamber cine MR imaging was used for analysis. The left ventricular end diastole phase was chosen after manual review in SA, and 2-, 3- and 4-chamber views. The left ventricular range was defined using 2 basal points and 1 apical point, the endocardial and epicardial contours were drawn automatically in 2- and 4-chamber view sand each SA slice, and the observer needed to confirm and make necessary adjustments manually if the contouring was not exact. Then, LV global and regional strain parameters were computed automatically. Global strain parameters included global radial strain (GRS), global circumferential strain (GCS), and global longitudinal strain (GLS); and regional radial strain, regional circumferential strain, and regional longitudinal strain were computed according to the AHA 16-segment model (Figures 1,2). If the computed borders were not satisfactory, the contour was adjusted, and the analysis was repeated. Finally, RRS, RCS, and the regional longitudinal strain of hypertrophic segments (RLS) were calculated for further analysis.

\section{Reproducibility}

Intraobserver and interobserver agreement for global strain and mean regional strain was tested in a subset of 10 healthy volunteers and $10 \mathrm{HCM}$ patients who were selected randomly. Both observers had more than 5 years' experience in CMR diagnosis and post-processing. One observer 

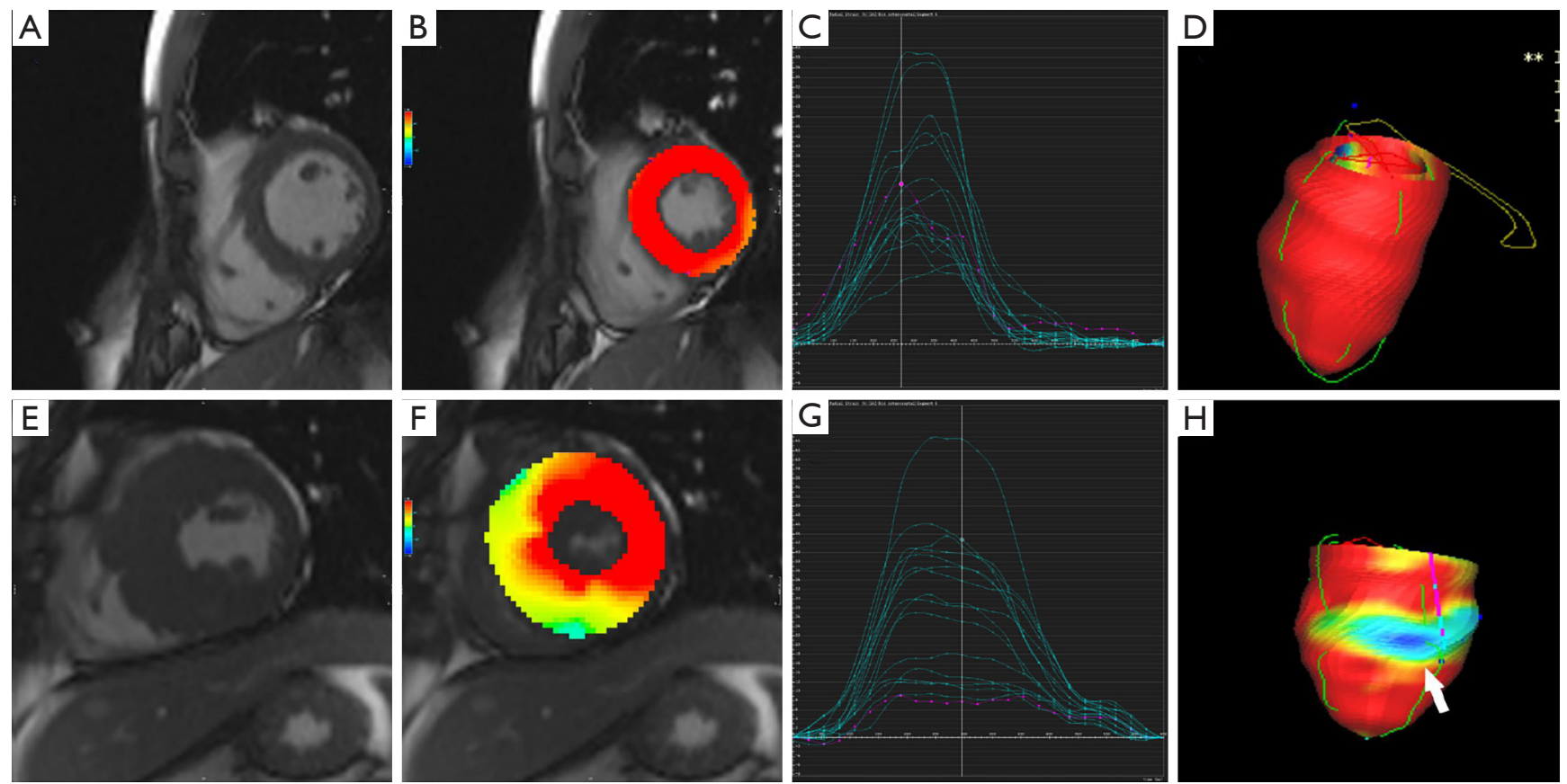

Figure 1 Feature tracking analysis in a healthy volunteer and a hypertrophic cardiomyopathy (HCM) patient. Feature tracking analysis in one healthy volunteer: left ventricular wall thickness (A) and regional radial strain of segments (B) in the mid-left ventricle were normal. (C) Shows regional radial strain curves of American Heart Association (AHA) segments. (D) Shows 3D model of radial strain. Feature tracking analysis in one HCM patient, where septal, anterior, and inferior wall (E) were thickened, and regional radial strain of thickened segments (F) were decreased. The purple curve shows the regional radial strain of the mid anteroseptal wall (G). Regional radial strain curves of AHA segments $(\mathrm{H})$ in a $3 \mathrm{D}$ model show the regional radial strain of the mid anteroseptal wall (white arrow).
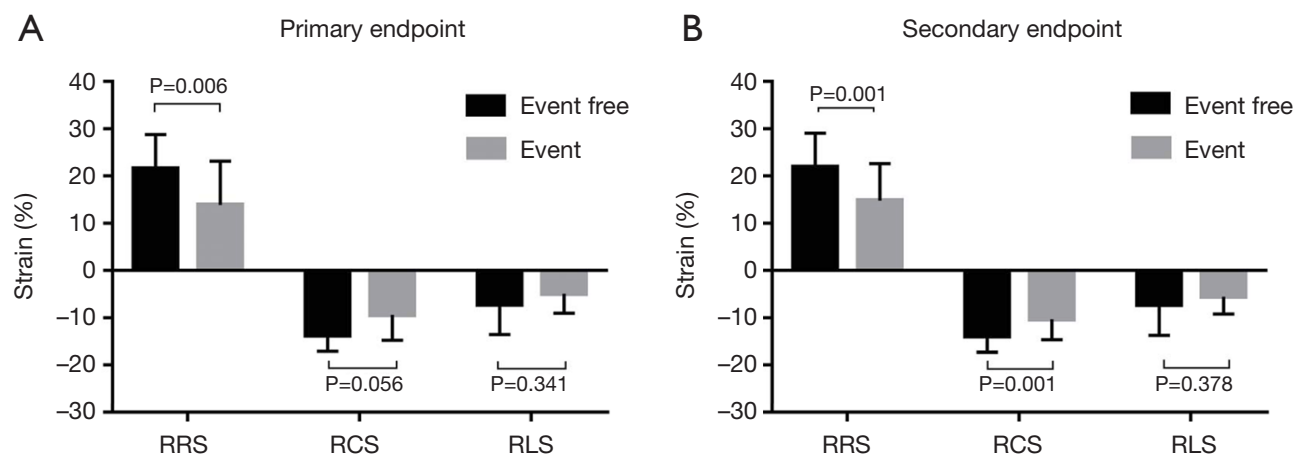

Figure 2 Bar graph of groups for primary and secondary end points. For primary end points (A), the difference of regional radial strain of hypertrophic segments (RRS) between the event and event-free groups was statistically significant. For secondary end points (B), the differences in RRS and regional circumferential strain of hypertrophic segments between groups were statistically significant. 
performed feature tracking analysis, which was repeated after four weeks for intraobserver reliability, and the other observer, who was blind to the myocardium contour and results of the first observer, performed feature tracking analysis on the same participants.

\section{Clinical outcomes}

Patient outcome data were obtained via medical electronic records, direct communication, or telephone interviews with patients or their family members. The initial time was the day of the first CMR examination, and the end of follow-up time was November 2019. The primary end points was defined as all-cause mortality or implantable cardioverter-defibrillator (ICD) discharge due to ventricular fibrillation of tachycardia, and the secondary end points was a combination of primary end points and hospitalization due to progression of heart failure. CMR measurements and feature tracking analysis were blind to outcome data.

\section{Statistical analyses}

The quantitative data are presented as mean $\pm S D$, and categorical data are presented as frequencies and percentages. The Kolmogorov-Smirnov test was used to assess the distribution of data. The independent samples Student's $t$ test was used to compare normally distributed data between groups, and the Mann-Whitney U test was used for nonnormally distributed data. The chi-squared or Fisher's exact test was used to analyze categorical variables. Pearson's or Spearman's correlations were used to perform bivariate correlation analysis for normally and nonnormally distributed data, respectively. Intraclass correlation coefficients (ICC) for inter- and intraobserver agreement were used to test the reproducibility of strain indices. Univariate and multivariate Cox proportional hazards regression analysis was used to calculate the hazard ratio (HR) and $95 \%$ confidence interval (CI). We examined the proportionality assumption by assessing interactions between each variable and follow-up time. Kaplan-Meier survival curve analysis was performed, and the log-rank method was used to compare groups. For primary and secondary end points, the optimal cutoff values of CMR parameters were determined according to the area under the curve (AUC) of receiver operating characteristic (ROC) curve analysis. Statistical analysis was performed using SPSS (version 20.0; IBM Corp., Armonk, NY, USA), GraphPad Prism (version 7.00; GraphPad Software, Inc., San Diego, CA, USA) and MedCalc (version 18.2.1; MedCalc Software Ltd., Ostend, Belgium). All variables with a $\mathrm{P}$ value $<0.05$ were considered statistically significant.

\section{Results}

\section{Baseline characteristics}

In all, $104 \mathrm{HCM}$ patients and 30 healthy volunteers were enrolled in this study. Demographic data and baseline characteristics are shown in Table 1. In the $104 \mathrm{HCM}$ patients, $20(19.2 \%)$ had obstructive HCM, and the remaining 84 (80.8\%) had non-obstructive HCM, including 17 patients $(16.4 \%)$ with apical HCM, 4 (3.9\%) with

Table 1 Baseline characteristics and cardiovascular magnetic resonance indices in healthy control and hypertrophic cardiomyopathy patients

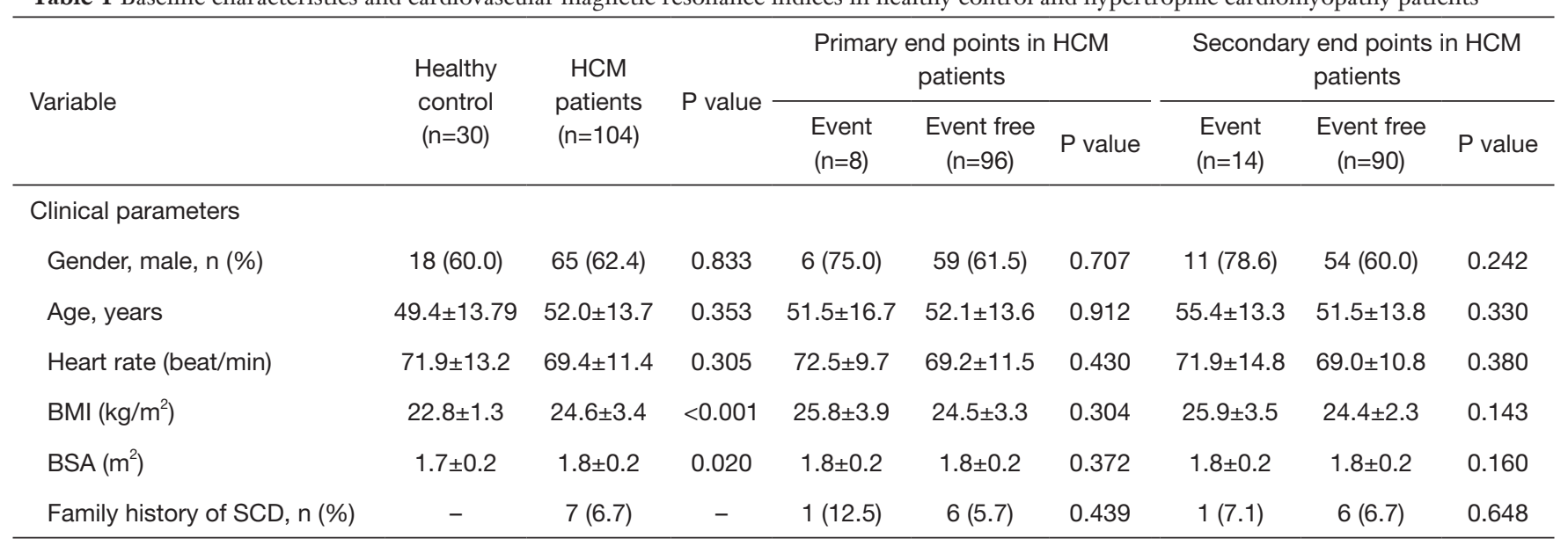

Table 1 (continued) 
Table 1 (continued)

\begin{tabular}{|c|c|c|c|c|c|c|c|c|c|}
\hline \multirow{2}{*}{ Variable } & \multirow{2}{*}{$\begin{array}{l}\text { Healthy } \\
\text { control } \\
(n=30)\end{array}$} & \multirow{2}{*}{$\begin{array}{c}\text { HCM } \\
\text { patients } \\
(n=104)\end{array}$} & \multirow{2}{*}{$P$ value } & \multicolumn{3}{|c|}{$\begin{array}{l}\text { Primary end points in } \mathrm{HCM} \\
\text { patients }\end{array}$} & \multicolumn{3}{|c|}{$\begin{array}{l}\text { Secondary end points in HCM } \\
\text { patients }\end{array}$} \\
\hline & & & & $\begin{array}{l}\text { Event } \\
(\mathrm{n}=8)\end{array}$ & $\begin{array}{l}\text { Event free } \\
\qquad(n=96)\end{array}$ & $P$ value & $\begin{array}{l}\text { Event } \\
(n=14)\end{array}$ & $\begin{array}{l}\text { Event free } \\
\quad(n=90)\end{array}$ & $P$ value \\
\hline History of syncope, n (\%) & - & $13(12.5)$ & - & $3(37.5)$ & $10(10.4)$ & 0.060 & $4(28.6)$ & $9(10.0)$ & 0.072 \\
\hline Diabetes mellitus, n (\%) & - & $16(15.4)$ & - & $1(12.5)$ & $15(15.6)$ & 1.000 & $2(14.3)$ & $14(15.6)$ & 1.000 \\
\hline Hypertension, n (\%) & - & $35(33.7)$ & - & $1(12.5)$ & $34(35.4)$ & 0.262 & $6(42.9)$ & $29(32.2)$ & 0.545 \\
\hline NYHA stage & - & $1.3 \pm 0.6$ & - & $1.4 \pm 0.7$ & $1.2 \pm 0.6$ & 0.385 & $1.2 \pm 0.6$ & $1.2 \pm 0.6$ & 0.981 \\
\hline Obstructive HCM, n (\%) & - & $20(19.8)$ & - & $3(37.5)$ & $17(17.7)$ & 0.179 & $4(28.6)$ & $16(17.8)$ & 0.464 \\
\hline Elevated troponin, $\mathrm{n}(\%)$ & - & $28(26.9)$ & - & $2(25.0)$ & $26(27.1)$ & 1.000 & $4(28.6)$ & $24(26.7)$ & 1.000 \\
\hline \multicolumn{10}{|l|}{ CMR parameters } \\
\hline LVWT (mm) & $8.3 \pm 1.1$ & $21.8 \pm 5.0$ & $<0.001$ & $25.4 \pm 5.8$ & $21.5 \pm 4.9$ & 0.048 & $24.6 \pm 5.1$ & $21.4 \pm 4.9$ & 0.017 \\
\hline LVEDV (mL) & $118.5 \pm 18.3$ & $140.9 \pm 31.3$ & $<0.001$ & $147.5 \pm 30.0$ & $140.3 \pm 31.5$ & 0.533 & $155.5 \pm 29.1$ & $138.6 \pm 31.1$ & 0.059 \\
\hline LVESV (mL) & $40.5 \pm 8.4$ & $51.4 \pm 19.5$ & 0.035 & $63.4 \pm 17.8$ & $50.4 \pm 19.4$ & 0.069 & $68.2 \pm 20.7$ & $48.7 \pm 18.1$ & $<0.001$ \\
\hline LVEF (\%) & $65.8 \pm 5.2$ & $63.7 \pm 10.8$ & 0.465 & $55.4 \pm 15.9$ & $64.4 \pm 10.1$ & 0.118 & $55.2 \pm 14.9$ & $65.0 \pm 9.5$ & 0.019 \\
\hline Mass (g) & $72.8 \pm 18.1$ & $142.9 \pm 49.0$ & $<0.001$ & $170.4 \pm 58.7$ & $140.7 \pm 47.7$ & 0.099 & $178.7 \pm 49.9$ & $137.4 \pm 46.7$ & 0.003 \\
\hline RVEDV (mL) & $117.9 \pm 19.5$ & $106.5 \pm 28.9$ & 0.014 & $89.9 \pm 16.1$ & $107.9 \pm 29.4$ & 0.090 & $100.2 \pm 23.3$ & $107.5 \pm 29.7$ & 0.382 \\
\hline LGE (g) & - & $12.7 \pm 18.2$ & - & $21.0 \pm 14.2$ & $12.0 \pm 18.4$ & 0.028 & $29.5 \pm 27.1$ & $10.1 \pm 15.0$ & $<0.001$ \\
\hline GRS (\%) & $37.7 \pm 9.8$ & $27.8 \pm 11.1$ & $<0.001$ & $15.5 \pm 7.5$ & $28.8 \pm 10.7$ & 0.001 & $17.4 \pm 10.8$ & $29.4 \pm 10.3$ & $<0.001$ \\
\hline GCS (\%) & $-20.7 \pm 2.5$ & $-18.0 \pm 4.4$ & $<0.001$ & $-12.4 \pm 3.7$ & $-18.9 \pm 4.0$ & $<0.001$ & $-13.5 \pm 3.8$ & $-19.2 \pm 3.9$ & $<0.001$ \\
\hline GLS (\%) & $-13.2 \pm 2.1$ & $-8.9 \pm 3.2$ & $<0.001$ & $-6.1 \pm 1.3$ & $-9.2 \pm 3.2$ & 0.009 & $-5.6 \pm 1.6$ & $-9.4 \pm 3.1$ & $<0.001$ \\
\hline RRS (\%) & - & $20.9 \pm 7.6$ & - & $13.9 \pm 9.2$ & $21.5 \pm 7.2$ & 0.006 & $14.8 \pm 7.8$ & $21.9 \pm 7.2$ & 0.001 \\
\hline RCS (\%) & - & $-13.4 \pm 3.7$ & - & $-9.3 \pm 5.4$ & $-13.8 \pm 3.4$ & 0.056 & $-10.3 \pm 4.4$ & $-13.9 \pm 3.4$ & 0.001 \\
\hline RLS (\%) & - & $-7.0 \pm 6.3$ & - & $-5.0 \pm 4.1$ & $-7.2 \pm 6.4$ & 0.341 & $-5.6 \pm 3.6$ & $-7.2 \pm 6.6$ & 0.378 \\
\hline
\end{tabular}

HMC, hypertrophic cardiomyopathy; BMI, body mass index; BSA, body surface area; SCD, sudden cardiac death; NYHA, New York Heart Association; BNP, brain natriuretic peptide; LVWT, LV wall thickness; LVEDV, LV end diastolic volume; LVESV, LV end systolic volume; LVEF, LV ejection fraction; RVEDV, RV end diastolic volume; RVESV, RV end systolic volume; RVEF, RV ejection fraction; LAV, left atrial volume; LAEF, left atrial ejection fraction; LGE, late gadolinium enhancement; GRS, global radial strain; GCS, global circumferential strain; GLS, global longitudinal strain; RRS, regional radial strain of hypertrophic segments; RCS, regional circumferential strain of hypertrophic segments; RLS, regional longitudinal strain of hypertrophic segments. 
Table 2 Correlation matrix between regional strain of hypertrophic segments and other cardiovascular magnetic resonance parameters

\begin{tabular}{|c|c|c|c|c|c|c|}
\hline Variable & \multicolumn{2}{|c|}{ RRS (\%) } & \multicolumn{2}{|c|}{ RCS (\%) } & \multicolumn{2}{|c|}{ RLS (\%) } \\
\hline LVWT (mm) & -0.431 & $<0.001$ & 0.435 & $<0.001$ & 0.222 & 0.024 \\
\hline LVEDV (mL) & -0.116 & 0.241 & 0.069 & 0.488 & -0.221 & 0.024 \\
\hline LVESV (mL) & -0.215 & 0.029 & 0.149 & 0.131 & -0.071 & 0.473 \\
\hline Mass (g) & -0.342 & $<0.001$ & 0.321 & 0.001 & -0.052 & 0.597 \\
\hline RVEDV (mL) & 0.116 & 0.241 & -0.158 & 0.110 & -0.276 & 0.005 \\
\hline RVESV (mL) & 0.091 & 0.359 & -0.139 & 0.160 & -0.238 & 0.015 \\
\hline RVEF (\%) & 0.070 & 0.479 & -0.034 & 0.731 & 0.036 & 0.717 \\
\hline LAEF (\%) & 0.058 & 0.558 & -0.045 & 0.649 & -0.091 & 0.359 \\
\hline LGE (g) & -0.457 & $<0.001$ & 0.439 & $<0.001$ & 0.221 & 0.024 \\
\hline GRS (\%) & 0.677 & $<0.001$ & -0.646 & $<0.001$ & -0.183 & 0.063 \\
\hline GCS (\%) & -0.717 & $<0.001$ & 0.687 & $<0.001$ & 0.211 & 0.032 \\
\hline GLS (\%) & -0.517 & $<0.001$ & 0.495 & $<0.001$ & 0.177 & 0.073 \\
\hline
\end{tabular}

R, correlation coefficient; LVWT, LV wall thickness; LVEDV, LV end diastolic volume; LVESV, LV end systolic volume; LVEF, LV ejection fraction; RVEDV, RV end diastolic volume; RVESV, RV end systolic volume; RVEF, RV ejection fraction; LAV, left atrial volume; LAEF, left atrial ejection fraction; LGE, late gadolinium enhancement; GRS, global radial strain; GCS, global circumferential strain; GLS, global longitudinal strain; RRS, regional radial strain of hypertrophic segments; RCS, regional circumferential strain of hypertrophic segments; RLS, regional longitudinal strain of hypertrophic segments.

concentric HCM, and 63 (60.6\%) with septal HCM. There were 433 hypertrophic segments in 104 patients and $4.2 \pm 1.9$ hypertrophic segments per patient according to the AHA 16-segment model. HCM patients showed an increased body mass index (BMI) and body surface area (BSA) compared with healthy volunteers.

\section{CMR indices between groups}

For CMR parameters, the chambers of LV, RV, and LA were dilated in HCM patients, and LAEF was reduced in the HCM group (Table 1). However, LVEF and RVEF between HCM volunteers and healthy patients did not reach statistical significance. Feature tracking analysis indicated impaired global strain (GRS, GCS, and GLS). The regional strain of hypertrophic segments (RRS, RCS, and RLS) were also impaired compared with mean regional strain of all segments in the healthy control group $(20.9 \% \pm 7.6 \%$ vs. $37.4 \% \pm 7.3 \%,-13.4 \% \pm 3.7 \%$ vs. $-20.1 \% \pm 2.2 \%$,
$-7.0 \% \pm 6.3 \%$ vs. $-15.7 \% \pm 2.8 \%$, respectively).

\section{Correlations between regional strain of hypertrophic segments and other CMR indices in HCM patients}

For regional strain of hypertrophic segments (Table 2), bivariate analysis indicated statistically significant correlations between RRS and LVWT, LVESV, LVEF, mass, LGE, GRS, GCS, and GLS. Correlations between RCS and LVWT, LVEF, mass, LGE, GRS, GCS, and GLS; and correlations between RLS and LVWT, LVEDV, RVEDV, RVESV, LGE, and GCS were also statistically significant. RRS was most strongly correlated with GCS $(\mathrm{r}=-0.72)$, RCS was most strongly correlated with GCS $(r=0.69)$, and RLS correlated with other indices only weakly.

\section{Outcomes}

The median follow-up time was 32 months, and during the 
follow-up time, $8(7.7 \%)$ patients reached a primary end points, 2 of whom (1.9\%) reached all-cause death; 6 (5.8\%) patients experienced ICD discharge due to ventricular arrhythmia; and 14 (5.8\%) patients reached a secondary end points and were admitted to hospital due to heart failure.

For the primary end points group, the event group had a thicker wall and LGE compared with the event-free group, but the difference of total LV mass and other LV, RV, and LA structural and functional parameters between the two groups was not statistically significant. The event group also showed worse global strain (GRS, GCS, and GLS) and regional strain of hypertrophic segments (RRS and RCS) except RLS.
HCM patients with secondary end points presented greater LGE; increased LVWT, mass, and LVESV; and worse LVEF, RVEF, LAEF, global strain, RRS, and RCS compared with HCM patients without secondary end points (Figure 3).

\section{Univariate and multivariate analyses}

In univariate Cox regression analysis (Table 3), history of syncope, LVWT, LVESV, LVEF, GRS, GCS, RRS, and RCS were associated with the primary and secondary end points. GCS (HR 1.58, 95\% CI: 1.02-2.44), RRS (HR 1.64, 95\% CI: 1.13-2.38), and RCS (HR 2.35, 95\% CI:
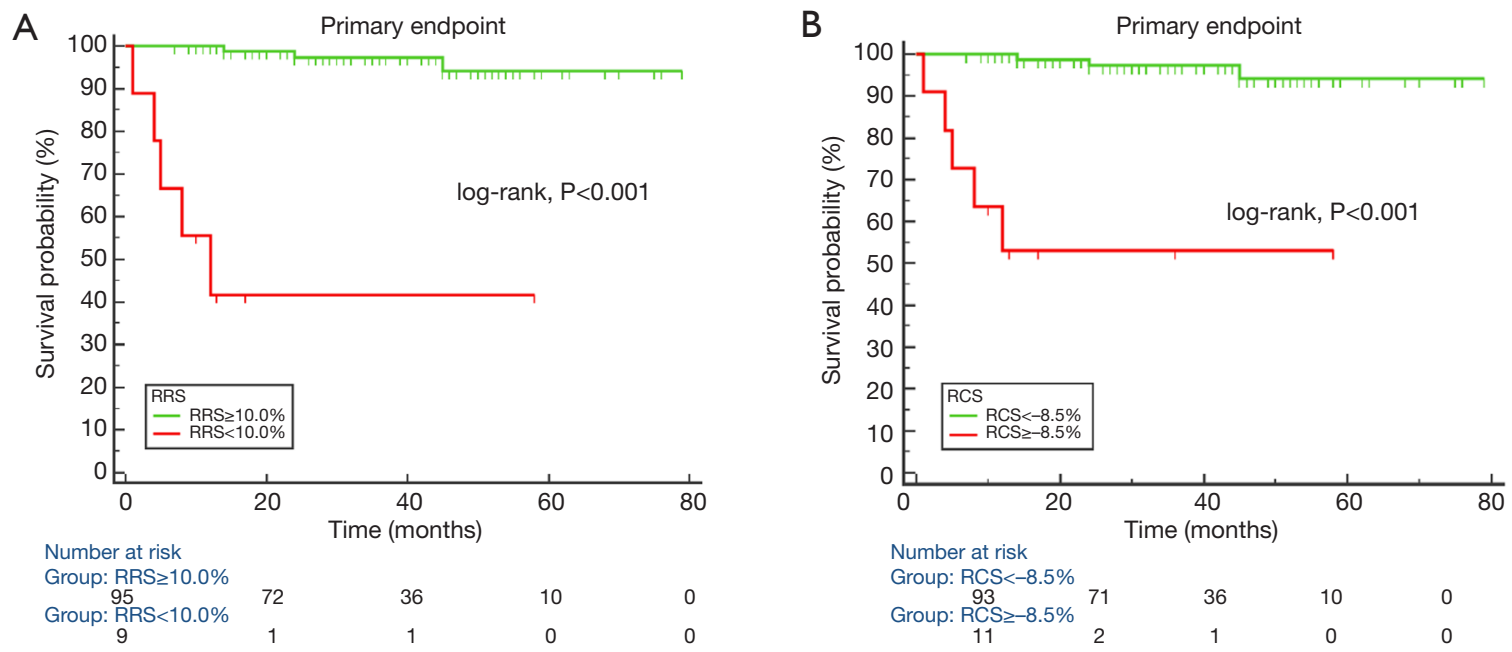

Figure 3 Primary end points Kaplan-Meier survival curves for groups of hypertrophic cardiomyopathy (HCM) patients. Primary end points Kaplan-Meier survival analysis for groups of HCM patients with different regional radial strain of hypertrophic segments (cutoff value was $9.97 \%$ ) (A) and regional circumferential strain of hypertrophic segments (cutoff value was $-8.46 \%$ ) (B).

Table 3 Univariate Cox regression analysis of all hypertrophic cardiomyopathy patients for primary and secondary end points

\begin{tabular}{|c|c|c|c|c|c|c|}
\hline Variable & \multicolumn{3}{|c|}{ Primary end points $(n=8)$} & \multicolumn{3}{|c|}{ Secondary end points $(n=14)$} \\
\hline \multicolumn{7}{|l|}{ Clinical parameters } \\
\hline Gender, n & 1.63 & $0.33-8.13$ & 0.552 & 2.10 & $0.58-7.54$ & 0.257 \\
\hline Age, years & 1.00 & $0.95-1.06$ & 0.917 & 1.02 & $0.98-1.07$ & 0.250 \\
\hline BMI $\left(\mathrm{kg} / \mathrm{m}^{2}\right)$ & 1.12 & $0.91-1.37$ & 0.290 & 1.12 & $0.96-1.31$ & 0.155 \\
\hline $\operatorname{BSA}\left(m^{2}\right)$ & 1.16 & $0.81-1.67$ & 0.410 & 1.20 & $0.91-1.58$ & 0.196 \\
\hline Family history of SCD, $\mathrm{n}$ & 2.06 & $0.26-16.84$ & 0.496 & 1.13 & $0.15-8.66$ & 0.905 \\
\hline
\end{tabular}

Table 3 (continued) 
Table 3 (continued)

\begin{tabular}{|c|c|c|c|c|c|c|}
\hline Variable & \multicolumn{3}{|c|}{ Primary end points $(n=8)$} & \multicolumn{3}{|c|}{ Secondary end points $(n=14)$} \\
\hline History of syncope, $n$ & 4.69 & $1.11-19.75$ & 0.035 & 3.26 & $1.02-10.42$ & 0.047 \\
\hline Diabetes mellitus, $\mathrm{n}$ & 0.82 & $0.10-6.69$ & 0.855 & 0.94 & $0.21-4.18$ & 0.930 \\
\hline Coronary heart disease, $\mathrm{n}$ & 1.64 & $0.33-8.17$ & 0.549 & 1.88 & $0.59-6.03$ & 0.286 \\
\hline \multicolumn{7}{|l|}{ NYHA stage } \\
\hline $\mathrm{NYHA}=2$ & 1.87 & $2.23-15.60$ & 0.563 & 0.96 & $0.12-7.36$ & 0.966 \\
\hline $\mathrm{NYHA}=3$ & 2.51 & $0.30-21.04$ & 0.396 & 1.15 & $0.16-8.89$ & 0.894 \\
\hline Obstructive HCM, n & 2.51 & $0.60-10.53$ & 0.209 & 1.69 & $0.53-5.40$ & 0.376 \\
\hline Atrial fibrillation, $\mathrm{n}$ & 2.55 & $0.31-20.87$ & 0.382 & 2.89 & $0.64-12.93$ & 0.166 \\
\hline \multicolumn{7}{|l|}{ CMR parameters } \\
\hline LVWT (mm) & 1.13 & $1.01-1.27$ & 0.037 & 1.10 & $1.01-1.21$ & 0.023 \\
\hline LVEDV (mL) & 1.01 & $0.99-1.03$ & 0.530 & 1.01 & $1.00-1.03$ & 0.069 \\
\hline LVESV (mL) & 1.03 & $1.00-1.06$ & 0.040 & 1.04 & $1.02-1.06$ & $0 . .001$ \\
\hline LVEF (\%) & 0.94 & $0.90-0.99$ & 0.012 & 0.94 & $0.91-0.98$ & 0.001 \\
\hline Mass (g) & 1.01 & $1.00-1.02$ & 0.080 & 1.01 & $1.01-1.02$ & 0.003 \\
\hline RVEDV (mL) & 0.97 & $0.94-1.00$ & 0.080 & 0.99 & $0.97-1.01$ & 0.333 \\
\hline LGE (g) & 1.02 & $0.99-1.04$ & 0.177 & 1.02 & $1.01-1.04$ & 0.001 \\
\hline GRS (\%) & 0.84 & $0.78-0.93$ & 0.001 & 0.88 & $0.82-0.94$ & $<0.001$ \\
\hline GCS (\%) & 1.53 & $1.21-1.94$ & $<0.001$ & 1.34 & $1.17-1.54$ & $<0.001$ \\
\hline GLS (\%) & 1.18 & $1.05-1.34$ & 0.008 & 1.21 & $1.11-1.33$ & $<0.001$ \\
\hline RRS (\%) & 0.82 & $0.72-0.93$ & 0.003 & 0.85 & $0.77-0.93$ & $<0.001$ \\
\hline RCS (\%) & 1.45 & $1.17-1.79$ & 0.001 & 1.33 & $1.14-1.55$ & $<0.001$ \\
\hline RLS (\%) & 1.05 & $0.96-1.15$ & 0.280 & 1.04 & $0.96-1.12$ & 0.331 \\
\hline
\end{tabular}

$\mathrm{Cl}$, confidence interval; HR, hazard ratio; BMI, body mass index; BSA, body surface area; SCD, sudden cardiac death; NYHA, New York Heart Association; BNP, brain natriuretic peptide; LVWT, LV wall thickness; LVEDV, LV end diastolic volume; LVESV, LV end systolic volume; LVEF, LV ejection fraction; RVEDV, RV end diastolic volume; RVESV, RV end systolic volume; RVEF, RV ejection fraction; LAV, left atrial volume; LAEF, left atrial ejection fraction; LGE, late gadolinium enhancement; GRS, global radial strain; GCS, global circumferential strain; GLS, global longitudinal strain; RRS, regional radial strain of hypertrophic segments; RCS, regional circumferential strain of hypertrophic segments; RLS, regional longitudinal strain of hypertrophic segments. 
Table 4 Multivariate Cox regression analysis of all hypertrophic cardiomyopathy patients for primary end points

\begin{tabular}{lccc}
\hline \multirow{2}{*}{ Variable } & \multicolumn{3}{c}{ Multivariate } \\
\cline { 2 - 4 } & $\mathrm{HR}$ & $95 \% \mathrm{Cl}$ & P value \\
\hline History of syncope, $\mathrm{n}$ & 2.29 & $0.26-20.04$ & 0.455 \\
LVWT (mm) & 1.04 & $0.87-1.24$ & 0.687 \\
LVESV (mL) & 0.94 & $0.86-1.03$ & 0.165 \\
LVEF (\%) & 0.89 & $0.77-1.01$ & 0.071 \\
GRS (\%) & 0.93 & $0.77-1.11$ & 0.389 \\
GCS (\%) & 1.58 & $1.02-2.44$ & 0.039 \\
GLS (\%) & 1.09 & $0.86-1.38$ & 0.475 \\
RRS (\%) & 1.64 & $1.13-2.38$ & 0.010 \\
RCS (\%) & 2.35 & $1.20-4.59$ & 0.012 \\
\hline
\end{tabular}

LVWT, LV wall thickness; LVESV, LV end systolic volume; LVEF, LV ejection fraction; GRS, global radial strain; GCS, global circumferential strain; GLS, global longitudinal strain; RRS, regional radial strain of hypertrophic segments; RCS, regional circumferential strain of hypertrophic segments.

1.20-4.59) were still independent predictors of primary end points in multivariate Cox regression analysis for primary end points (Table 4); meanwhile, for secondary end points, LAEF (HR 0.88, 95\% CI: 0.79-0.99), GLS (HR 1.31, 95\% CI: 1.01-1.69), RRS (HR 1.71, 95\% CI: 1.09-2.66), and RCS (HR 2.63, 95\% CI: 1.20-5.75) remained independent predictors (Table 5).

\section{Kaplan-Meier survival analysis}

Table 6 shows the diagnostic performance of GCS, RRS, and RCS for primary end points patients and event-free patients; and of LAEF, GLS, RRS, and RCS for secondary end points patients and event-free patients. RRS and RCS showed similar diagnostic performance for primary end points patients and event-free patients, and for secondary end points patients and event-free patients, respectively. Figure 4 shows the Kaplan-Meier survival curves for primary and secondary end points event-free survival. Patients with RRS $<10.0 \%$ and RCS $\geq-8.5 \%$ had higher rates of primary end points, while patients with RRS $<17.9 \%$ and RCS $\geq-12.1 \%$ experienced higher rates of secondary end points.

\section{Inter- and intraobserver agreement}

Inter- and intraobserver agreement for strain parameters
Table 5 Multivariate Cox regression analysis of all hypertrophic cardiomyopathy patients for secondary end points

\begin{tabular}{|c|c|c|c|}
\hline \multirow{2}{*}{ Variable } & \multicolumn{3}{|c|}{ Multivariate } \\
\hline & $\mathrm{HR}$ & $95 \% \mathrm{Cl}$ & $P$ value \\
\hline History of syncope, $n$ & 1.14 & $0.15-8.47$ & 0.898 \\
\hline LVWT (mm) & 1.17 & $0.96-1.42$ & 0.118 \\
\hline LVESV (mL) & 1.04 & $0.96-1.13$ & 0.357 \\
\hline LVEF (\%) & 1.00 & $0.88-1.13$ & 0.952 \\
\hline Mass (g) & 0.99 & $0.96-1.01$ & 0.305 \\
\hline RVEF (\%) & 1.06 & $0.96-1.16$ & 0.291 \\
\hline $\mathrm{LAV}_{\text {min }}(\mathrm{mL})$ & 0.96 & $0.91-1.01$ & 0.095 \\
\hline LAEF (\%) & 0.88 & $0.79-0.99$ & 0.030 \\
\hline LGE (g) & 1.03 & $0.99-1.06$ & 0.132 \\
\hline GRS (\%) & 0.98 & $0.84-1.13$ & 0.745 \\
\hline GCS (\%) & 1.30 & $0.96-1.77$ & 0.092 \\
\hline GLS (\%) & 1.31 & $1.01-1.69$ & 0.041 \\
\hline RRS (\%) & 1.71 & $1.09-2.66$ & 0.019 \\
\hline RCS (\%) & 2.63 & $1.20-5.75$ & 0.016 \\
\hline
\end{tabular}

LVWT, LV wall thickness; LVESV, LV end systolic volume; LVEF, LV ejection fraction; RVEF, RV ejection fraction; LAV, left atrial volume; LAEF, left atrial ejection fraction; LGE, late gadolinium enhancement; GRS, global radial strain; GCS, global circumferential strain; GLS, global longitudinal strain; RRS, regional radial strain of hypertrophic segments; $\mathrm{RCS}$, regional circumferential strain of hypertrophic segments.

was good. GRS, GCS, and GLS showed excellent intraobserver $(0.953,0.949$, and 0.937 , respectively) and interobserver ICC $(0.949,0.910$, and 0.907 , respectively) agreement, while RRS, RCS, and RLS also showed good intraobserver $(0.931,0.913$, and 0.902 , respectively) and interobserver agreement $(0.926,0.893$, and 0.891 , respectively).

\section{Discussion}

CMR strain parameters indicated impaired global strain, although LVEF and RVEF were preserved, which has been reported by previous studies $(14,22)$. The majority of HCM patients in this study had New York Hear Association (NYHA) stage I or II diagnoses, and normal LVEF but reduced GRS, GCS, and GLS, revealing subclinical ventricular dysfunction. These results show the value of strain detecting early ventricular dysfunction. There were 
Table 6 Receiver operating characteristic curves analysis for event and event-free patients

\begin{tabular}{lccccc}
\hline Variables & AUC & Cut-off value (\%) & Sensitivity (\%) & Specificity (\%) & Median survival time (months) \\
\hline GCS1 & 0.87 & -12.9 & 75.0 & 89.6 & 13.5 \\
GLS2 & 0.92 & -6.4 & 78.6 & 94.4 & 11.5 \\
LAEF2 & 0.72 & 51.1 & 85.7 & 60.0 & 28 \\
RRS1 & 0.76 & 10.0 & 63.5 & 98.8 & 10 \\
RRS2 & 0.75 & 17.9 & 71.4 & 71.1 & 27 \\
RCS1 & 0.75 & -8.5 & 62.5 & 94.8 & 12 \\
RCS2 & 0.75 & -12.1 & 71.4 & 74.4 & 18 \\
\hline
\end{tabular}

1, for patients with primary end points and event-free patients; 2, for patients with secondary end points and event-free patients. AUC, area under the curve; GCS, global circumferential strain; GLS, global longitudinal strain; RRS, regional radial strain of hypertrophic segments; RCS, regional circumferential strain of hypertrophic segments.
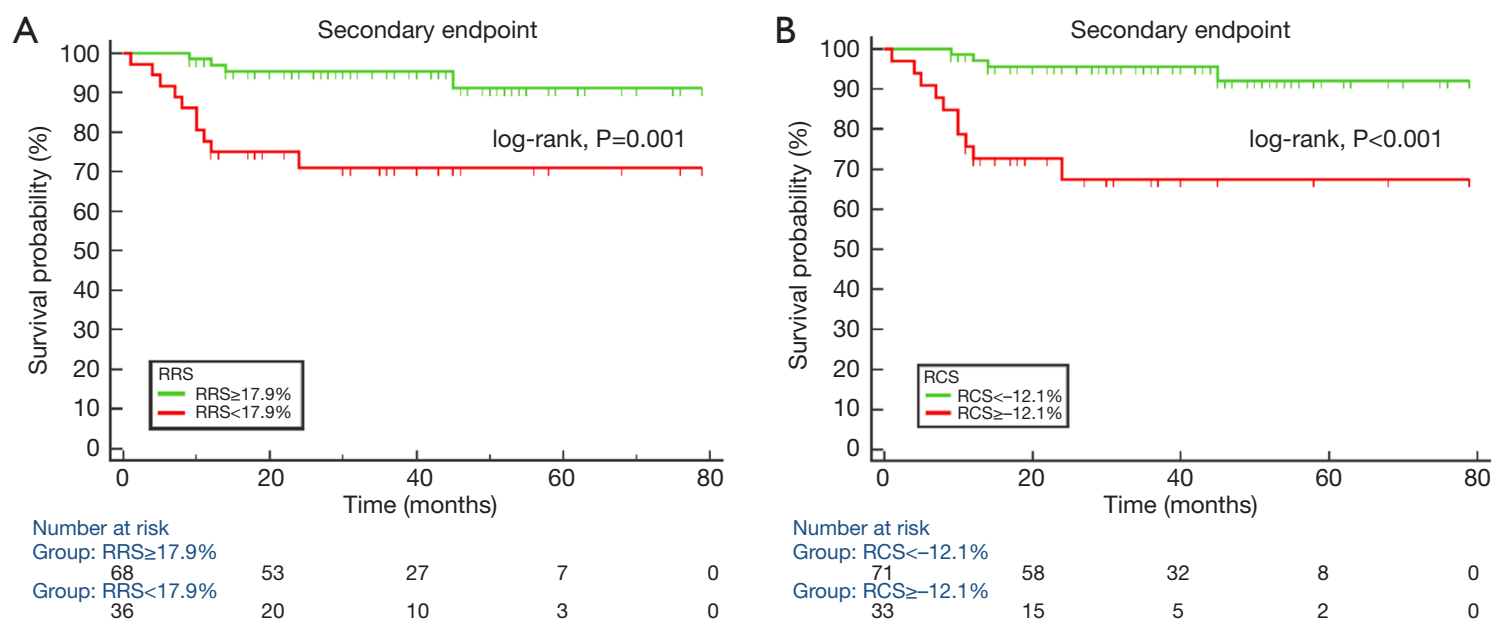

Figure 4 Kaplan-Meier survival curves of secondary end points for groups of hypertrophic cardiomyopathy (HCM) patients. Secondary end points Kaplan-Meier survival analysis for groups of HCM patients with different regional radial strain of hypertrophic segments (cut-off value was $17.93 \%$ ) (A) and regional circumferential strain of hypertrophic segments (cutoff value was $-12.1 \%$ ) (B).

hypertrophic and non-hypertrophic segments in HCM, and regional function varied between different segments. Therefore, regional strain was be better than global strain in assessing regional function, including each segment in all slices or AHA 16 segments, and the evaluation of regional dysfunction by regional strain was more accurate and detailed in HCM patients. Comparison of regional strain showed that the regional strain of hypertrophic segments was lower than the mean regional strain in the control group. These results may be related to the underlying regional histopathology of HCM (23). Moreover, for preclinical HCM patients with normal LVWT, LV diastolic function was reduced in participants with preclinical HCM compared with control participants (24), but the variable degree of diastolic dysfunction may be related to variable global and regional function. Reduced LA function and enlarged LA volume suggest that impaired LV diastolic function was possibly prevalent and led to LA structural and functional abnormalities in this study, which is also supported by previous studies $(10,25,26)$.

Another recent study (9) reported increased LGE and reduced $\mathrm{LV}$ strain in $\mathrm{HCM}$ patients with adverse outcomes. In our study, both LVWT and LGE were increased more in the primary and secondary end points group, and was accompanied by reduced global strain and RRS. Moreover, biventricular and LA ejection fraction were all reduced 
in HCM patients with primary end points. Our study showed not only LGE and global strain abnormalities but also abnormal regional strain. Regional radial strain of hypertrophic segments and RCS were reduced in HCM patients with secondary end points, but the difference in RLS between groups for primary and secondary end points did not reach statistical significance. The possible reason for this may be that RRS and RCS were generated by SA view cine MR imaging, and RLS was calculated by 2-, 3-, and 4-chamber view cine MR imaging; SA view was a stack of images covering the whole $\mathrm{LV}$; while longitudinal view cine MR imaging's coverage was part of $\mathrm{LV}$, and this could miss a portion of the hypertrophic segments.

A previous study (27) found global strain to be related to LV mass and LGE. Other studies $(17,18)$ on regional strain of HCM patients reported regional strain to be correlated with LVWT, LGE, and LVEF. In our study, all regional strain of hypertrophic segments correlated with LVWT, LGE, and GCS, but the coefficients were relatively small between RLS and other CMR indices. On the other hand, RRS and RCS correlated with LVWT, LGE, and all global strains more strongly, while RRS and RCS strongly correlated with GCS ( $\mathrm{r}=0.717$ and 0.687 , respectively). Abnormal pathology of myocardial hypertrophy possibly contributed to impaired LV diastolic filling and chamber stiffness. This indicates that RRS and RCS were more robust and stable than RLS in HCM patients.

While decreased global strain and regional strain in HCM patients compared with normal volunteers has been demonstrated $(18)$, other reports $(10,16,28)$ indicate that HCM patients with adverse outcomes have worse global strain. However, the regional strain's change between event and event-free group is unknown. In this study, we further explored the performance of regional strain of hypertrophic segments in HCM patients for primary and secondary end points. The comparison between groups showed statistically significant regional strain except RLS. Regional radial strain of hypertrophic segments and RCS were reduced more in HCM patients for secondary end points, while RRS remained impaired in HCM patients for primary end points. These changes of regional strain may help to provide more information on segmental myocardial functional abnormalities in HCM patients with different outcomes.

Previous studies (28-30) demonstrated global ventricular function including LVEF and RVEF, and global strains were useful for further risk assessment of life-threatening cardiovascular events. In this study, history of syncope,
LVWT, LVESV, LVEF, GRS, GCS, RRS, and RCS were associated with primary end points, history of syncope, LVWT, LVESV, LVEF, and mass. In univariate analysis, RVEF, LAVmax, LAEF, LGE, GRS, GCS, GLS, RRS, and RCS were associated with secondary end points. Multivariate Cox regression analysis further demonstrated GCS, RRS, and RCS to be predictors for primary end points, and LAEF, GLS, RRS, and RCS remained independent predictors for secondary end points. Previous studies have also demonstrated that global strain is a predictor for adverse outcomes in HCM patients $(28,29)$. Hinojar et al. (29) have also reported left atrial ejection fraction as a predictor for adverse outcome in HCM patients with poor cardiac outcomes, particularly cardiovascular mortality and HF. Although global strain is a potential predictor for major adverse cardiovascular outcome, the prognostic value of regional strain by CMR feature tracking imaging is still unclear. This study demonstrated RRS and RCS were independent predictors for both primary and secondary end points. Kaplan-Meier survival analysis for primary and secondary end points events indicated the cutoff value of RRS for primary end points event was lower than those with secondary end points events, and the cutoff value of RCS for primary end points events was higher than the value for the secondary end points events, which indicates worse regional radial and circumferential strain of the hypertrophic segments in HCM patients with primary end points events. It is reasonable to assume that severely damaged regional strain was related to malignant results such as SCD or ICD discharge. Estimation of major adverse cardiovascular events risk in HCM patients was an integral part of clinical management, and these 2 indices may potentially have predictive value in clinical risk assessment.

Currently, LVEF is still the reference standard of ventricular function despite its limitations in regional function assessment. Myocardial strain is a superior parameter to evaluate global and regional myocardial function. Usually, speckle tracking echocardiography, CMR tagging, and feature tracking are used to perform strain analysis (31). CMR feature tracking imaging is currently widely available due to measurement of myocardial deformation without the need for dedicated acquisition and complex postprocessing. Global strain not only has good agreement with CMR tagging but also demonstrates good reproducibility $(15,32,33)$. Inter- and intraobserver agreement for strain parameters were also good in this study. The limitation of CMR feature tracking imaging is mainly the potentially lower temporal resolution compared 
with speckle tracking echography and the fact that the strain value varies between different methods and software.

This study has some limitations. First, it was a singlecenter study, the sample size was not sufficiently large, and the number of adverse events in patients was relatively small. Therefore, the statistical power of this study was limited. Second, several types of software were used for CMR feature tracking strain analysis, and strain value and reproducibility might therefore have varied depending on the method and software used. Third, among the 104 HCM patients, the majority underwent CMR examination using a 1.5 T MRI scanner, and 12 patients underwent examination using a 3.0 T system. Although the cine MR imaging's frame was the same between different scanners, the signal to noise ratio of images by $3.0 \mathrm{~T}$ was superior, which might have improved the myocardial contouring, and this may also have had potential effects on strain results. Finally, HCM was diagnosed clinically and not through use of biopsy or a genetic test, and thus no preclinical HCM patients were included. Therefore, the prognostic performance of regional strain and other CMR indices for preclinical HCM patients remain unknown and should be addressed by future research.

In summary, HCM patients with primary and secondary end points demonstrated more thickened walls and LGE, along with worse global strain and RRS. Regional radial strain of hypertrophic segments and RCS were associated with primary and secondary end points in univariate analysis and remained independent significant predictors in multivariate analysis. HCM patients with lower RRS and RCS experienced a higher rate of adverse cardiovascular outcomes.

\section{Acknowledgments}

We thank Professor Paul Schoenhagen for his assistance with language and copyediting.

Funding: This study was funded by a key project grant from the Jinhua Municipal Science and Technology Bureau (No. 2021-3-107) and a key project grant from the Jinhua Municipal Central Hospital (No. JY2019-1-02).

\section{Footnote}

Conflicts of Interest: All authors have completed the ICMJE uniform disclosure form (available at https://dx.doi. org/10.21037/qims-21-42). XC, JP, XZ, LY, LC, and YH are current employees of the Affiliated Jinhua Hospital,
Zhejiang University School of Medicine. RY is a current employee of The Second Affiliated Hospital, Zhejiang University School of Medicine. JS is a current employee of Jinhua People's Hospital. The other authors have no conflicts of interest to declare.

Ethical Statement: The authors are accountable for all aspects of the work in ensuring that questions related to the accuracy or integrity of any part of the work are appropriately investigated and resolved. The study was conducted in accordance with the Declaration of Helsinki (as revised in 2013). The study was approved by the ethics committee of our institute, and written informed consent was obtained from each patient and volunteer.

Open Access Statement: This is an Open Access article distributed in accordance with the Creative Commons Attribution-NonCommercial-NoDerivs 4.0 International License (CC BY-NC-ND 4.0), which permits the noncommercial replication and distribution of the article with the strict proviso that no changes or edits are made and the original work is properly cited (including links to both the formal publication through the relevant DOI and the license). See: https://creativecommons.org/licenses/by-nc-nd/4.0/.

\section{References}

1. Elliott PM, Anastasakis A, Borger MA, Borggrefe M, Cecchi F, et al. 2014 ESC Guidelines on diagnosis and management of hypertrophic cardiomyopathy: the Task Force for the Diagnosis and Management of Hypertrophic Cardiomyopathy of the European Society of Cardiology (ESC). Eur Heart J 2014;35:2733-79.

2. Semsarian C, Ingles J, Maron MS, Maron BJ. New perspectives on the prevalence of hypertrophic cardiomyopathy. J Am Coll Cardiol 2015;65:1249-54.

3. Gersh BJ, Maron BJ, Bonow RO, Dearani JA, Fifer MA, Link MS, Naidu SS, Nishimura RA, Ommen SR, Rakowski H, Seidman CE, Towbin JA, Udelson JE, Yancy CW; American College of Cardiology Foundation/ American Heart Association Task Force on Practice Guidelines. 2011 ACCF/AHA Guideline for the Diagnosis and Treatment of Hypertrophic Cardiomyopathy: a report of the American College of Cardiology Foundation/ American Heart Association Task Force on Practice Guidelines. Developed in collaboration with the American Association for Thoracic Surgery, American Society of Echocardiography, American Society of Nuclear 
Cardiology, Heart Failure Society of America, Heart Rhythm Society, Society for Cardiovascular Angiography and Interventions, and Society of Thoracic Surgeons. J Am Coll Cardiol 2011;58:e212-60.

4. Baxi AJ, Restrepo CS, Vargas D, Marmol-Velez A, Ocazionez D, Murillo H. Hypertrophic Cardiomyopathy from A to Z: Genetics, Pathophysiology, Imaging, and Management. Radiographics 2016;36:335-54.

5. Elliott PM, Gimeno JR, Thaman R, Shah J, Ward D, Dickie S, Tome Esteban MT, McKenna WJ. Historical trends in reported survival rates in patients with hypertrophic cardiomyopathy. Heart 2006;92:785-91.

6. Kubo T, Gimeno JR, Bahl A, Steffensen U, Steffensen M, Osman E, Thaman R, Mogensen J, Elliott PM, Doi Y, McKenna WJ. Prevalence, clinical significance, and genetic basis of hypertrophic cardiomyopathy with restrictive phenotype. J Am Coll Cardiol 2007;49:2419-26.

7. Maron MS, Finley JJ, Bos JM, Hauser TH, Manning WJ, Haas TS, Lesser JR, Udelson JE, Ackerman MJ, Maron BJ. Prevalence, clinical significance, and natural history of left ventricular apical aneurysms in hypertrophic cardiomyopathy. Circulation 2008;118:1541-9.

8. Guttmann OP, Rahman MS, O'Mahony C, Anastasakis A, Elliott PM. Atrial fibrillation and thromboembolism in patients with hypertrophic cardiomyopathy: systematic review. Heart 2014;100:465-72.

9. Weng Z, Yao J, Chan RH, He J, Yang X, Zhou Y, He Y. Prognostic Value of LGE-CMR in HCM: A MetaAnalysis. JACC Cardiovasc Imaging 2016;9:1392-402.

10. Hinojar R, Zamorano JL, Fernández-Méndez M, Esteban A, Plaza-Martin M, González-Gómez A, Carbonell A, Rincón LM, Nácher JJJ, Fernández-Golfín C. Prognostic value of left atrial function by cardiovascular magnetic resonance feature tracking in hypertrophic cardiomyopathy. Int J Cardiovasc Imaging 2019;35:1055-65.

11. Green JJ, Berger JS, Kramer CM, Salerno M. Prognostic value of late gadolinium enhancement in clinical outcomes for hypertrophic cardiomyopathy. JACC Cardiovasc Imaging 2012;5:370-7.

12. Vigneault DM, Yang E, Jensen PJ, Tee MW, Farhad H, Chu L, Noble JA, Day SM, Colan SD, Russell MW, Towbin J, Sherrid MV, Canter CE, Shi L, Ho CY, Bluemke DA. Left Ventricular Strain Is Abnormal in Preclinical and Overt Hypertrophic Cardiomyopathy: Cardiac MR Feature Tracking. Radiology 2019;290:640-8.

13. Wu XP, Li YD, Wang YD, Zhang M, Zhu WW, Cai QZ, Jiang W, Sun LL, Ding XY, Ye XG, Qin YY, Jiang Z, Guo DC, Lu XZ. Decreased biventricular mechanics and functional reserve in nonobstructive hypertrophic cardiomyopathy patients: implications for exercise capacity. Int J Cardiovasc Imaging 2019;35:869-79.

14. Neisius U, Myerson L, Fahmy AS, Nakamori S, ElRewaidy H, Joshi G, Duan C, Manning WJ, Nezafat R. Cardiovascular magnetic resonance feature tracking strain analysis for discrimination between hypertensive heart disease and hypertrophic cardiomyopathy. PLoS One 2019;14:e0221061.

15. Taylor RJ, Moody WE, Umar F, Edwards NC, Taylor TJ, Stegemann B, Townend JN, Hor KN, Steeds RP, Mazur W, Leyva F. Myocardial strain measurement with featuretracking cardiovascular magnetic resonance: normal values. Eur Heart J Cardiovasc Imaging 2015;16:871-81.

16. Tower-Rader A, Mohananey D, To A, Lever HM, Popovic ZB, Desai MY. Prognostic Value of Global Longitudinal Strain in Hypertrophic Cardiomyopathy: A Systematic Review of Existing Literature. JACC Cardiovasc Imaging 2019;12:1930-42.

17. Urbano-Moral JA, Rowin EJ, Maron MS, Crean A, Pandian NG. Investigation of global and regional myocardial mechanics with 3-dimensional speckle tracking echocardiography and relations to hypertrophy and fibrosis in hypertrophic cardiomyopathy. Circ Cardiovasc Imaging 2014;7:11-9.

18. Xu HY, Chen J, Yang ZG, Li R, Shi K, Zhang Q, Liu X, Xie LJ, Jiang L, Guo YK. Early marker of regional left ventricular deformation in patients with hypertrophic cardiomyopathy evaluated by MRI tissue tracking: The effects of myocardial hypertrophy and fibrosis. J Magn Reson Imaging 2017;46:1368-76.

19. Schulz-Menger J, Bluemke DA, Bremerich J, Flamm SD, Fogel MA, Friedrich MG, Kim RJ, von KnobelsdorffBrenkenhoff F, Kramer CM, Pennell DJ, Plein S, Nagel E. Standardized image interpretation and post-processing in cardiovascular magnetic resonance - 2020 update : Society for Cardiovascular Magnetic Resonance (SCMR): Board of Trustees Task Force on Standardized Post-Processing. J Cardiovasc Magn Reson 2020;22:19.

20. Hudsmith LE, Petersen SE, Francis JM, Robson MD, Neubauer S. Normal human left and right ventricular and left atrial dimensions using steady state free precession magnetic resonance imaging. J Cardiovasc Magn Reson 2005;7:775-82.

21. Harrigan CJ, Peters DC, Gibson CM, Maron BJ, Manning WJ, Maron MS, Appelbaum E. Hypertrophic cardiomyopathy: quantification of late gadolinium enhancement with contrast-enhanced cardiovascular MR 
imaging. Radiology 2011;258:128-33.

22. Ozawa K, Funabashi N, Takaoka H, Kamata T, Kanaeda A, Saito M, Nomura F, Kobayashi Y. Characteristic myocardial strain identified in hypertrophic cardiomyopathy subjects with preserved left ventricular ejection fraction using a novel multi-layer transthoracic echocardiography technique. Int J Cardiol 2015;184:237-43.

23. Camici PG, Olivotto I, Rimoldi OE. The coronary circulation and blood flow in left ventricular hypertrophy. J Mol Cell Cardiol 2012;52:857-64.

24. Ho CY, Carlsen C, Thune JJ, Havndrup O, Bundgaard H, Farrohi F, Rivero J, Cirino AL, Andersen PS, Christiansen M, Maron BJ, Orav EJ, Kober L. Echocardiographic strain imaging to assess early and late consequences of sarcomere mutations in hypertrophic cardiomyopathy. Circ Cardiovasc Genet 2009;2:314-21.

25. Maron BJ, Haas TS, Maron MS, Lesser JR, Browning JA, Chan RH, Olivotto I, Garberich RF, Schwartz RS. Left atrial remodeling in hypertrophic cardiomyopathy and susceptibility markers for atrial fibrillation identified by cardiovascular magnetic resonance. Am J Cardiol 2014;113:1394-400.

26. Yang Y, Yin G, Jiang Y, Song L, Zhao S, Lu M. Quantification of left atrial function in patients with nonobstructive hypertrophic cardiomyopathy by cardiovascular magnetic resonance feature tracking imaging: a feasibility and reproducibility study. J Cardiovasc Magn Reson 2020;22:1.

27. Shi RY, An DA, Chen BH, Wu R, Wu CW, Du L, Zhu J, Jiang M, Xu JR, Wu LM. High T2-weighted signal intensity is associated with myocardial deformation in hypertrophic cardiomyopathy. Sci Rep 2019;9:2644.

28. Yang F, Wang J, Li Y, Li W, Xu Y, Wan K, Sun J, Han Y, Chen Y. The prognostic value of biventricular long axis

Cite this article as: Chen $\mathrm{X}$, Pan J, Shu J, Zhang X, Ye L, Chen L, Hu Y, Yu R. Prognostic value of regional strain by cardiovascular magnetic resonance feature tracking in hypertrophic cardiomyopathy. Quant Imaging Med Surg 2022;12(1):627-641. doi: 10.21037/qims-21-42 strain using standard cardiovascular magnetic resonance imaging in patients with hypertrophic cardiomyopathy. Int J Cardiol 2019;294:43-9.

29. Hinojar R, Fernández-Golfín C, González-Gómez A, Rincón LM, Plaza-Martin M, Casas E, GarcíaMartín A, Fernandez-Mendez MA, Esteban A, Nacher JJJ, Zamorano JL. Prognostic implications of global myocardial mechanics in hypertrophic cardiomyopathy by cardiovascular magnetic resonance feature tracking. Relations to left ventricular hypertrophy and fibrosis. Int J Cardiol 2017;249:467-72.

30. Hen Y, Iguchi N, Kanisawa M, Takada K, Machida H, Takara A, Teraoka K, Takamisawa I, Takayama $\mathrm{M}$, Yoshikawa T. Additive prognostic significance of ejection fraction for ESC risk model in hypertrophic cardiomyopathy patients. Heart Vessels 2020;35:391-8.

31. Amzulescu MS, De Craene M, Langet H, Pasquet A, Vancraeynest D, Pouleur AC, Vanoverschelde JL, Gerber BL. Myocardial strain imaging: review of general principles, validation, and sources of discrepancies. Eur Heart J Cardiovasc Imaging 2019;20:605-19.

32. Augustine D, Lewandowski AJ, Lazdam M, Rai A, Francis J, Myerson S, Noble A, Becher H, Neubauer S, Petersen SE, Leeson P. Global and regional left ventricular myocardial deformation measures by magnetic resonance feature tracking in healthy volunteers: comparison with tagging and relevance of gender. J Cardiovasc Magn Reson 2013;15:8.

33. Zhu C, Wang S, Wang S, Meng Y, Yang Q, Nie C, Sun $H$. Prevalence and characteristics of intramural coronary artery in hypertrophic obstructive cardiomyopathy: a coronary computed tomography and invasive angiography study. Quant Imaging Med Surg 2021;11:162-71. 